\title{
THE AGE STRUCTURE OF POPULATION-DEPENDENT GENERAL BRANCHING PROCESSES IN ENVIRONMENTS WITH A HIGH CARRYING CAPACITY
}

\author{
HAMZA, K., JAGERS, P., AND KLEBANER, F. C.
}

\begin{abstract}
The age structure of populations supercritical below and subcritical above a carrying capacity is investigated, the result being a law of large numbers, as the capacity increases and time passes, provided the starting population is not little.
\end{abstract}

\section{Preliminaries and Assumptions}

General branching processes describe populations where individuals live, reproduce, and die clonally and independently according to any fixed pattern allowing random variation between individuals, [8]. They can be rendered even more general by allowing life-spans and reproduction to be influenced by population size, through population and age dependent hazard and birth rates. (Not to complicate matters, we assume continuous life-spans and reproductions. The time discrete case is parallell.) In [13] the size of such populations was studied, the parameter $K$ referring to a threshhold or carrying capacity, above which the population is too large to sustain itself: if it is larger than $K$, reproduction turns subcritical.

Thus, consider a hazard rate $h_{z}^{K}(u)$ of a $u$-aged individual in a population of size $z$. At death the individual might split into a random number of offspring, again depending upon both population size and mother's age then. Write $m_{z}^{K}(u)$ and $v_{z}^{K}(u)$ for the mean and second moment of this offspring at splitting of a $u$-aged individual in a $z$-sized population. (The latter will of course be zero for higher organisms, which do not reproduce by splitting, like plants and animals, but not so for cells and bacteria, and physical particles.) Finally, there are age-specific population dependent birth rates, describing how higher organism individuals give birth during life. What unites the resulting processes and renders them amenable to analysis is that they are Markovian in the age structure, [10] p. 208.

At little further cost all rates involved can be assumed to depend also upon the age-distribution, i.e. vector of all ages present in the population, $A=\left(a_{1}, a_{2}, \ldots a_{z}\right)$ rather than just its size $z$. Here we study the composition of such populations, notably the age-distribution.

Intuitively, it is clear that a population starting large must shrink to the neighbourhood of the carrying capacity, and also that a population starting small either dies out quickly or else increases up to around the carrying capacity. It is populations starting from levels close to $K$, or somehow having arrived there, that may display interesting features during their finite but long persistance, [13].

Here we assume that the demographic parameters $h_{A}^{K}(u), b_{A}^{K}(u), m_{A}^{K}(u)$, and (for some results) $v_{A}^{K}(u)$ are all uniformly bounded, i.e. $\sup _{K, A, u} b_{A}^{K}(u)<\infty$ etc. Further they are supposed to be continuous and indeed Lipschitz in a strong sense, to be made precise in Definition 3.1. (Actually, boundedness of the death rate is an unnecessarily strong requirement.) A framework satisfying these conditions will be loosely referred to as having a smooth demography.

A particular case to bear in mind is that where demographic parameters are in fact determined by the population density $x=z / K=|A| / K,|A|=(1, A)=z$, if $A=\left(a_{1}, a_{2}, \ldots a_{z}\right)$, so that

All three authors acknowledge support from the Australian Research Council under Discovery Project 1201022728, Stochastic Populations: Theory and Applications. One of them (PJ) thanks B. A. Sevastyanov for introducing him into research on branching processes. 
they can be written like $b_{x}(u)$ etc. for short. Demographic smoothness would then refer to the dependence upon $x$. A showcase for this is binary splitting with the splitting probability $K /(K+z)=1 /(1+x)$, no births during life and the same life span distribution throughout, unaffected by population circumstances, [22].

Michaelis-Menten kinetics, [11], and describes a situation where the population is supercritical below the carrying capacity $K$, critical at, and sub-critical above $K$.

Population processes with size dependence were first analysed for Galton-Watson style processes, $[18,19,20]$. Birth-and-death style process have been investigated in a series of papers by Méléard, Champagnat, Lambert, and coauthors, [2, 3, 4]. More general processes were treated in $[12,25]$ and [13]. The present paper follows up the latter by studying the complete age-distribution.

In a population with carrying capacity $K$, the ages of the individuals constitute a finite integer-valued measure on $\mathbb{R}^{+}$, hereafter denoted $A^{K}$. The purpose of this work is to establish the law of large numbers for $A^{K} / K$ as $K$ approaches infinity.

Similar problems have recently been treated independently in [23]. That work has a broader scope in that individuals also have types that can change by mutation, and which may affect the population dynamics. It is more specific in its assumptions about how the parameter corresponding to the carrying capacity affects individual life and reproduction, viz. assuming that birth and death intensities have the same leading term, proportional to the carrying capacity, Méléard and Tran also assume that interaction between individuals is additive and does not affect reproduction. The paper is written in the birth-and-death tradition, thus not allowing reproduction by splitting.

\section{Age and Population Dependent Processes. Foundations}

This section contains definitions and basic properties to be used subsequently. Essentially it consists in writing down the generator of our measure-valued Markov process, and an integral representation, known as Dynkin's formula. For a function $f$ on $\mathbb{R}^{+}$and $A \in \mathcal{M}\left(\mathbb{R}^{+}\right)$, the space of finite, positive measures on $\mathbb{R}^{+}$, the following notation is used:

$$
(f, A)=\int f(u) A(d u)=\sum_{i=1}^{z} f\left(a_{j}\right),|A|=(1, A),
$$

the middle equality subsuming that $A$ is discrete with the mass points $a_{j}, j=1,2, \ldots z$. We often simply write $z=|A|$. The population process evolving as described is denoted $A^{K}=\left\{A_{t}^{K} ; t \geq 0\right\}$, $Z_{t}^{K}:=\left|A_{t}^{K}\right|, X_{t}^{K}:=Z_{t}^{K} / K$. The size and density dependent cases, respectively, are when parameters depend on $A_{t}^{K}$ only through $Z_{t}^{K}$ and when dependence on both $K$ and $A_{t}^{K}$ are channelled through $X_{t}^{K}$.

Suffixed entities $\mathbb{P}_{A}$ and $\mathbb{E}_{A}$ indicate that the population started at time $t=0$ not from one newborn ancestor but rather from $z$ individuals, of ages $A=\left(a_{1}, \ldots, a_{z}\right)$, respectively. No index means a start from a given age configuration, or a probability/expectation in a meaning clear from the context. Often the setup is the classical start from one newborn individual, $A_{0}^{K}=(0)$. In any case, we shall always assume without loss of generality (simply condition on $A_{0}^{K}$ ) that $A_{0}^{K}$ is deterministic. In this section we consider the population process for fixed $K$ and hence need not spell it out.

The generator of a Markov measure valued population and age dependent branching process was given in [12].

Theorem 2.1 ([12]). For a bounded differentiable function $F$ on $\mathbb{R}^{+}$and a continuously differentiable function $f$ on $\mathbb{R}^{+}$, the following limit exists

$$
\lim _{t \rightarrow 0} \frac{1}{t} \mathbb{E}_{A}\left\{F\left(\left(f, A_{t}\right)\right)-F((f, A))\right\}=\mathcal{G} F((f, A)),
$$


where

$$
\begin{aligned}
\mathcal{G} F((f, A))= & F^{\prime}((f, A))\left(f^{\prime}, A\right)+\sum_{j=1}^{z} b_{A}\left(a_{j}\right)\{F(f(0)+(f, A))-F((f, A))\}+ \\
& +\sum_{j=1}^{z} h_{A}\left(a_{j}\right)\left\{\mathbb{E}_{A}\left[F\left(Y\left(a^{j}\right) f(0)+(f, A)-f\left(a_{j}\right)\right)\right]-F((f, A))\right\},
\end{aligned}
$$

and $Y(a)$ denotes the number of children at death of a mother, dying at age a. Consequently, Dynkin's formula holds: for a bounded $C^{1}$-function $F$ on $\mathbb{R}\left(F \in C_{b}^{1}(\mathbb{R})\right)$ and a $C^{1}$-function $f$ on $\mathbb{R}^{+}$

$$
F\left(\left(f, A_{t}\right)\right)=F\left(\left(f, A_{0}\right)\right)+\int_{0}^{t} \mathcal{G} F\left(\left(f, A_{s}\right)\right) d s+M_{t}^{F, f}
$$

where $M_{t}^{F, f}$ is a local martingale with predictable quadratic variation

$$
\left\langle M^{F, f}, M^{F, f}\right\rangle_{t}=\int_{0}^{t} \mathcal{G} F^{2}\left(\left(f, A_{s}\right)\right) d s-2 \int_{0}^{t} F\left(\left(f, A_{s}\right)\right) \mathcal{G} F\left(\left(f, A_{s}\right)\right) d s .
$$

As a corollary the following representation was also obtained, see [12].

Theorem 2.2. For a $C^{1}$-function $f$ on $\mathbb{R}^{+}$

$$
\left(f, A_{t}\right)=\left(f, A_{0}\right)+\int_{0}^{t}\left(L_{A_{s}} f, A_{s}\right) d s+M_{t}^{f},
$$

where the linear operators $L_{A}$ are defined by

$$
L_{A} f=f^{\prime}-h_{A} f+f(0)\left(b_{A}+h_{A} m_{A}\right),
$$

and $M_{t}^{f}$ is a local square integrable martingale with the sharp bracket given by

$$
\left\langle M^{f}, M^{f}\right\rangle_{t}=\int_{0}^{t}\left(f^{2}(0) b_{A_{s}}+f^{2}(0) v_{A_{s}} h_{A_{s}}+h_{A_{s}} f^{2}-2 f(0) m_{A_{s}} h_{A_{s}} f, A_{s}\right) d s,
$$

In this $m_{A}(u)=\mathbb{E}_{A}[Y(u)]$ is the mean and $v_{A}(u)=\mathbb{E}_{A}\left[Y^{2}(u)\right]$ the second moment of the offspring-at-splitting distribution in a population with composition $A$. Subsequently, we shall apply this to situations with dependence also upon the carrying capacity $K$.

From Theorem 2.3 of [12] it follows that if a non-negative and bounded $f$ satisfies the (linear growth) condition (H1):

$$
\exists C>0 \text { s.t. } \forall A \in \mathcal{M}\left(\mathbb{R}^{+}\right),\left|\left(L_{A} f, A\right)\right| \leq C(1+f, A)
$$

where the constant $C$ may depend on $f$, then for any $A,\left(f, A_{t}\right)$ is integrable and its expectation is bounded,

$$
\mathbb{E}\left[\left(f, A_{t}\right)\right] \leq\left|A_{0}\right| U(t),
$$

for some increasing deterministic function $U$ that depends on $f$ alone. When $f=1, U$ can be chosen of the form $e^{C t}$. An argument for this and Proposition 2.1 is given in the Appendix.

Throughout the paper $C$ is used in a generic way to denote a constant whose value depends on the context and will generally depend on bounds of the demographic parameters and the function $f$, but not the age structure, $A$.

Moreover, $M_{t}^{f}$ is a true martingale. We say that a set of functions is uniformly bounded if the set of all function values is bounded. Note that $C$ will, in general, depend on $f$.

Proposition 2.1. If the birth and death intensities, $b_{A}, h_{A}, A \in \mathcal{M}\left(\mathbb{R}^{+}\right)$, and the mean at splitting $m_{A}, A \in \mathcal{M}\left(\mathbb{R}^{+}\right)$, are all uniformly bounded and the non-negative function $f$ and its derivative are bounded, then the growth condition (H1) is satisfied and the conclusion (2.7) holds. 
In particular, the function identically 1 satisfies condition (H1) so that

$$
\mathbb{E}\left[\left|A_{t}\right|\right] \leq\left|A_{0}\right| e^{C t} .
$$

Further, if the $v_{A}$ 's, $A \in \mathcal{M}\left(\mathbb{R}^{+}\right)$, are uniformly bounded, then $M_{t}^{f}$ is a square integrable martingale with quadratic variation

$$
\left\langle M^{f}, M^{f}\right\rangle_{t} \leq C \int_{0}^{t}\left|A_{s}\right| d s
$$

\section{Tightness And Fluid Approximation.}

Now we consider measure-valued processes $A^{K}=\left\{A_{t}^{K}, t \geq 0\right\}$ for different carrying capacities $K$. Parameters of the processes (the functions $h_{A^{K}}^{K}, b_{A^{K}}^{K}, m_{A^{K}}^{K}$ and sometimes $v_{A^{K}}^{K}$ ) may also depend upon $K$, in the manner outlined in the introduction. Thus, for fixed $K, A^{K}$ is a random function on $\mathbb{R}^{+}$with values in $\mathcal{M}\left(\mathbb{R}^{+}\right)$, the space of finite, positive measures on $\mathbb{R}^{+}$, equipped with its weak topology. We establish the weak convergence of the measure-valued processes $\left\{\frac{1}{K} A_{t}^{K}, t \geq 0\right\}_{K}$, writing $\bar{A}_{t}^{K}=A_{t}^{K} / K=\frac{1}{K} A_{t}^{K}$ interchangeably, in the Skorohod space $\mathbb{D}\left(\mathbb{R}^{+}, \mathcal{M}\left(\mathbb{R}^{+}\right)\right)$of all càdlàg functions from $\mathbb{R}^{+}$to $\mathcal{M}\left(\mathbb{R}^{+}\right)$. This is decomposed into several steps. First tightness is established. Then conditions are given for the limit of any convergent sequence to satisfy an integral equation, which has a unique solution.

Proposition 3.1. Assume that all demographic parameters are uniformly bounded and denote by $C_{g}$ and $C_{d}$ the constants that appear in (H1) and (2.9) respectively. Suppose also that the total mass of $\bar{A}_{0}^{K}$ is bounded, $D=\sup _{K}\left|\bar{A}_{0}^{K}\right|<\infty$, and fix an arbitrary $\varepsilon>0$. Then, for each $T, K \geq C_{g} T / \varepsilon$ and $a>(D+\varepsilon) e^{C_{g} T}$,

$$
\mathbb{P}\left(\sup _{t \leq T} \frac{1}{K}\left|A_{t}^{K}\right|>a\right) \leq \frac{C_{d} \varepsilon(D+\varepsilon)}{\left(a e^{-C_{g} T}-D-\varepsilon\right)^{2} C_{g} T}\left(1+\frac{1}{C_{g}} e^{C_{g} T}\right) .
$$

Theorem 3.1. Assume that all demographic parameters are uniformly bounded. Suppose also that the support of $\bar{A}_{0}^{K}$ and its total mass are bounded, $\sup _{K} \inf \left\{t>0: A_{0}^{K}((t,+\infty))=0\right\}<\infty$ and $D=\sup _{K}\left|\bar{A}_{0}^{K}\right|<\infty$. Then, the family $\left\{\bar{A}_{t}^{K}, t \geq 0\right\}_{K}$ is tight in $\mathbb{D}\left(\mathbb{R}^{+}, \mathcal{M}\left(\mathbb{R}^{+}\right)\right)$.

The next step is to make the concept of demographic smoothness precise. It comprises two conditions, where the first has already been relied upon.

Definition 3.1. A population process will be said to be demographically smoothly density dependent, or for short just demographically smooth, if:

C0 The model parameters, $b, h, m$, are uniformly bounded.

C1 They are also normed uniformly Lipschitz in the following sense: there is a $C>0$ such that for all $u$ and $K, \rho(\mu, \nu)$ denoting the Levy-Prokhorov distance between measures $\mu$ and $\nu$,

$$
\begin{aligned}
& \text { - }\left|b_{A}^{K}(u)-b_{B}^{K}(u)\right| \leq C \rho(A / K, B / K), \\
& \text { - }\left|h_{A}^{K}(u)-h_{B}^{K}(u)\right| \leq C \rho(A / K, B / K), \\
& \text { - }\left|m_{A}^{K}(u)-m_{B}^{K}(u)\right| \leq C \rho(A / K, B / K) .
\end{aligned}
$$

$\mathrm{C} 2 \bar{A}_{0}^{K} \Rightarrow \bar{A}_{0}^{\infty}$, and $\sup _{K}\left|\bar{A}_{0}^{K}\right|<\infty$. We say that the process stabilises initially.

Specifically, C1 is satisfied if there is a Lipschitz continuous function $m^{\infty}$ defined on $\mathcal{M}\left(\mathbb{R}^{+}\right)$, such that

$$
\begin{gathered}
m_{A}^{K}=m_{A / K}^{\infty}, \\
\left|m_{A}^{\infty}-m_{B}^{\infty}\right| \leq C_{L} \rho(A, B),
\end{gathered}
$$


and correspondingly functions $h^{\infty}$ and $b^{\infty}$ defined on $\mathcal{M}\left(\mathbb{R}^{+}\right) \times \mathbb{R}^{+}$which are Lipschitz continuous in $A$ uniformly in $u$, such that

$$
h_{A}^{K}(u)=h_{A / K}^{\infty}(u), \quad b_{A}^{K}(u)=b_{A / K}^{\infty}(u),
$$

and (with function norm being the sup norm, e.g. $\left\|h_{A}^{\infty}\right\|=\sup _{u \in \mathbb{R}^{+}} h_{A}^{\infty}(u)$ )

$$
\left\|h_{A}^{\infty}-h_{B}^{\infty}\right\| \leq C \rho(A, B),\left\|b_{A}^{\infty}-b_{B}^{\infty}\right\| \leq C \rho(A, B) .
$$

This form will typically enter in situations where we have density dependence, parameters governed by the age-composition through the population size normed by the carrying capacity.

Assumption $\mathrm{C} 1$ has the following consequence:

Proposition 3.2. If $\mu^{K}$ is a sequence of measures such that $\mu^{K} / K \Rightarrow \bar{\mu}^{\infty}$, then $m_{\bar{\mu}^{\infty}}^{\infty}:=$ $\lim _{K \rightarrow \infty} m_{\mu^{K}}^{K}$ exists and is a Lipschitz function of its argument. Similarly, $h_{\bar{\mu}^{\infty}}^{\infty}(u):=\lim _{K \rightarrow \infty} h_{\mu^{K}}^{K}(u)$ and $b_{\bar{\mu}^{\infty}}^{\infty}(u):=\lim _{K \rightarrow \infty} b_{\mu^{K}}^{K}(u)$ exist, and are Lipschitz functions of $\bar{\mu}^{\infty}$.

This will now be applied to the age distributions for various $t$ in a population process:

Theorem 3.2. In a demographically smoothly density dependent population process, the processes $\bar{A}^{K}$ converge weakly in the Skorokhod space $\mathbb{D}\left(\mathbb{R}^{+}, \mathcal{M}\left(\mathbb{R}^{+}\right)\right)$. The limiting measure-valued process, $\bar{A}^{\infty}$, displays no randomness, and for any test function $f, \bar{A}^{\infty}$ satisfies the integral equation

$$
\left(f, A_{t}\right)=\left(f, \bar{A}_{0}^{\infty}\right)+\int_{0}^{t}\left(L_{A_{s}}^{\infty} f, A_{s}\right) d s
$$

where $A_{t}$ is short for $\bar{A}_{t}^{\infty}$ and

$$
L_{A}^{\infty} f=f^{\prime}-h_{A}^{\infty} f+f(0)\left(b_{A}^{\infty}+h_{A}^{\infty} m_{A}^{\infty}\right) .
$$

Remark 1. Equation (3.1) is the weak form of the McKendrick-von Foerster equation for the density of $A_{t}, a(t, u)$

$$
\left(\frac{\partial}{\partial t}+\frac{\partial}{\partial u}\right) a(t, u)=-a(t, u) h_{A_{t}}(u), \quad a(t, 0)=\int_{0}^{\infty} m_{A_{t}}(u) h_{A_{t}}(u) a(t, u) d u .
$$

It can be obtained by integration by parts and the adjoint operator $L^{*}$,

$$
\left(f, A_{t}\right)=\left(f, A_{0}\right)+\int_{0}^{t}\left(f, L_{A_{s}}^{*} A_{s}\right) d s .
$$

Of course, smoothness of the density must also be proved.

This equation was obtained in [24] in a different setting.

Note. Once the unique existence of a time limiting age distribution with a density has been established, its form follows in the usual manner from the transport equation above, letting $t \rightarrow \infty$. The derivative of $a$ with respect to time vanishes in the limit, and $h_{t}(u) \rightarrow$ some $h(u)$, to be inserted in the limiting equation.

It is important to note, though, that the latter has a trivial null solution, if the population starts from a bounded number of ancestors, a mutant or a limited number of invaders, so that $\lim _{K \rightarrow \infty}\left|\bar{A}_{0}^{K}\right|=0$. Such populations either grow to reach a band around the carrying capacity or die out before that [13]. Growth, however, takes too long time to show up in the asympotics discussed in this section.

Indeed, as described in [13], under population size dependence and natural monotonicity assumptions (the larger the population, the smaller its rate of increase) a population from one or few invaders will evade early extinction with a probability bounded away from zero, and then grow to reach a level $d K, 0<d<1$ in time $T_{d}^{K}=O(\log K)$. Then it will linger around $K$ for a time that is exponential in $K, K \rightarrow \infty$. It is an interesting task to describe the asymptotic age distribtion at time $T_{d}^{K}$, as $K \rightarrow \infty$, and then the evolution of the process in a suitable 
evolutionary time scale that starts when the population enters a band around the carrying capacity, provided it so does. For results on this, in a setting partly more general and partly more special than ours, we refer to [23].

\section{REFERENCES}

[1] Billingsley P. Convergence of Probability Measures Second ed. (1999), New York etc.

[2] Champagnat N.. Ferrière R., and Méléard S. (2007) Individual-based probabilistic models of adaptive evolution and various scaling approximations. Progress in Probability 59 (2007), 75113, Birchhauser, Basel.

[3] Champagnat N.. Ferrière R., and Méléard, S. (2008) From individual stochastic processes to macroscopic models in adaptive evolution. Stochastic Models 24, 244.

[4] Champagnat N. and Lambert A. (2008) Adaptive dynamics in a logistic branching population. Banach Center Publ. 80, 235-244.

[5] Etheridge A. (2000) An Introduction to Superprocesses, Amer. Mathematical Society.

[6] Ethier S.N. and Kurtz T.G. (1986) Markov Processes. Wiley, London etc.

[7] Gorostiza L.G. and Lopez-Mimbela J.A. (1990) The multitype measure branching process. Adv. Appl. Prob. $22,49-67$.

[8] Haccou P., Jagers P., and Vatutin V. A. (2005) Branching Processes: Variation, Growth, and Extinction of Populations. Cambridge U. Press, Cambridge.

[9] Hoppensteadt F. (1975) Mathematial Theories of Populations: Demographics,, Genetics, and Epidemics. SIAM, Philadelphia.

[10] Jagers P. (1975) Branching Processes with Biological Applications. Wiley. London etc.

[11] Jagers P and Klebaner F.C. (2003) Random variation and concentration effects in PCR. Journal of Theoretical Biology 224, 299304.

[12] Jagers P and Klebaner F.C. (2000) Population-Size-Dependent and Age-Dependent Branching Processes. Stochastic Processes and Their Applications 87, 235-254.

[13] Jagers P. and Klebaner F.C. (2011) Population size dependent, age structured branching processes linger around their carrying capacity. J. Appl. Prob. 48A, 249-260.

[14] Jakubowski A. (1986) On the Skorokhod topology. Ann. Inst. H. Poincaré B22, 263-285.

[15] Joffe, A. and Metivier, M. (1986) Weak convergence of sequences of semimartingales with applications to multitype branching processes. Adv. Appl. Prob. 18, 20-65.

[16] Kallenberg O. (2002) Foundations of Modern Probability. 2nd Ed. Springer, Berlin etc.

[17] Kaspi H. and Ramanan K. Law of Large Numbers Limits for Many-Server Queues. (2011) Ann. Appl. Prob. 21(1), 33-114.

[18] Klebaner F.C. (1984) Geometric rate of growth in population size dependent branching processes. J. Appl. Prob. 21, 40-49.

[19] Klebaner F.C. (1989) Geometric growth in near-supercritical population size dependent multitype GaltonWatson processes. Ann. Prob. 17, 4, 1466-1477.

[20] Klebaner F.C. (1994) Asymptotic behaviour of Markov population processes with asymptotically linear rate of change. J. Appl. Prob. 31, 614-625.

[21] Klebaner F.C. Introduction to Stochastic Calculus with Applications. 3d Ed. Imperial College Press, 2012.

[22] Klebaner F. C., Sagitov S., Vatutin V.A., Haccou P, and Jagers P. (2011) Stochasticity in the adaptive dynamics of evolution: the bare bones. J. Biol. Dyn. 5, 147-162.

[23] Méléard S. and Tran V. C. (2012) Slow and fast scales for superprocess limits for age-structured populations. Stoch. Proc. Applic. 122, 250-276. G. Da Prato and L. Tubaro, Lecture Notes in Mathematics 1390, SpringerVerlag, Belin, 171-185.

[24] Oelschläger K. (1990) Limit theorems for age-structured populations. Ann. Prob. 18, 290-318.

[25] Tran, V. C. Large population limit and time behaviour of a stochastic particle model describing an-agestructured population. ESAIM: PS 12, 345-386 (2008)

School of Mathematical Sciences, Monash University, Clayton, Vic. 3058, Australia.

E-mail address: kais.hamza@monash.edu

Mathematical Sciences, Chalmers and University of Gothenburg, SE-412 96 Gothenburg, SweDEN.

E-mail address: jagers@chalmers.se

School of Mathematical Sciences, Monash University, Clayton, Vic. 3058, Australia.

E-mail address: fima.klebaner@monash.edu 